\title{
Psychometric Evaluation of the Stroke Impact Scale 3.0
}

\author{
Ercole Vellone, RN, MSN; Serenella Savini, RN, MSN; Roberta Fida, PhD; \\ Victoria Vaughan Dickson, PhD, RN; Gail D'Eramo Melkus, EdD, C-NP, FAAN; \\ Francisco Javier Carod-Artal, MD, PhD; Gennaro Rocco, RN, MSN; Rosaria Alvaro, RN, MSN
}

\begin{abstract}
Background: The Stroke Impact Scale 3.0 (SIS 3.0) is widely used to measure quality of life in stroke survivors; however, previous studies have not tested the original 8-factor structure of the scale. In addition, previous studies have shown floor and ceiling effect and weak reliability within the scale. Objective: The aim of this study was to evaluate the psychometric characteristics of the SIS 3.0, including its construct validity (factorial structure, concurrent and contrasting group validity), floor and ceiling effect, and reliability. Method: A cross-sectional design was used to study 392 stroke survivors enrolled in 16 rehabilitation facilities across Italy. Factorial structure of the SIS 3.0 was tested with confirmatory factor analysis. Concurrent and contrasting group validities were evaluated with other scales measuring functional capacities, neurological functions, cognition, anxiety, depression, and generic quality of life. Floor and ceiling effects were evaluated by determining the percentages of patients with the minimum and the maximum score at SIS 3.0. Reliability was determined by Cronbach's $\alpha$ and test-retest. Results: Participants were 71 years old on average (SD, 11 years); 55\% were men. Confirmatory factor analysis revealed a new 4-factor structure that fitted the data better than the original 8-factor structure did. Concurrent and contrasting group validity of the new 4-factor structure was supportive and no floor and ceiling effects were found. Internal consistency and test-retest reliability ranged between 0.79 and 0.98 . Conclusion: The new factorial structure of the SIS 3.0 with 4 factors showed better psychometric properties than the original 8-factor structure did. This evidence supports further use of the SIS 3.0 in clinical practice and research.
\end{abstract}

KEY WORDS: instrument validity and reliability, psychometrics, stroke survivors

Strokst troke represents a leading cause of death in industrialized countries with cardiovascular diseases and cancer and has a significant impact on individuals, families, and public health. ${ }^{1,2}$ Stroke is the second single most common cause of death, accounting for almost 1.1 million deaths in Europe each year, ${ }^{3}$ and in the United States, it is expected that nearly $4 \%$ of the population will have a stroke by $2030 .^{4}$

Stroke causes severe disabilities. One year after stroke, $30 \%$ of survivors complain of significant dis-

Ercole Vellone, RN, MSN

PhD Student, Research Fellow, Department of Biomedicine and Prevention, University of Rome Tor Vergata, Italy.

Serenella Savini, RN, MSN

PhD Student, Department of Biomedicine and Prevention, University of Rome Tor Vergata, Italy.

Roberta Fida, PhD

Assistant Professor, Department of Psychology, Sapienza University, Rome, Italy.

Victoria Vaughan Dickson, PhD, RN

Assistant Professor, College of Nursing, New York University.

Gail D'Eramo Melkus, EdD, C-NP, FAAN

Florence and William Downs Professor in Nursing Research, College of Nursing, New York University.

Francisco Javier Carod-Artal, MD, PhD

Visiting Professor of Neurology, Medicine and Health Sciences

Faculty, Universitat Internacional de Catalunya (UIC), Barcelona, ability, including limb spasticity or flaccidity, depression, cognitive impairment, disorientation, pain, malnutrition, and sleep disturbances. ${ }^{5}$ Because these problems influence the quality of life (QOL) of patients, it is important for clinicians and researchers to have measures that exhibit evidence of reliability and validity to evaluate the impact of stroke on QOL among survivors.

Until the late 1990s, generic tools were used to measure QOL in stroke survivors.

Spain, and Consultant Neurologist, Department of Neurology, Raigmore Hospital, Inverness, United Kingdom.

\section{Gennaro Rocco, RN, MSN}

Director, Center of Excellence for Nursing Scholarship, Rome, Italy.

Rosaria Alvaro, RN, MSN

Associate Professor, Department of Biomedicine and Prevention, University of Rome Tor Vergata, Italy.

This work was funded by the Center of Excellence for Nursing Scholarship, Rome, Italy.

The authors have no conflicts of interest to disclose.

\section{Correspondence}

Ercole Vellone, RN, MSN, Department of Biomedicine and Prevention, University of Rome Tor Vergata, Via Montpellier, 1 00133, Rome, Italy (ercole.vellone@uniroma2.it).

DOI: $10.1097 / \mathrm{JCN} .0000000000000145$ 
The most common instruments were the 36-item Short-Form Health Survey (SF-36), ${ }^{6}$ the Sickness Impact Profile, ${ }^{7}$ the Nottingham Health Profile, ${ }^{8}$ and the World Health Organization Quality of Life-Brief (WHOQOLBrief). ${ }^{9}$ These instruments assess only a few areas compromised by stroke and do not provide a comprehensive and specific assessment of stroke survivors' QOL.

In the last 2 decades, specific instruments have been developed to measure QOL in stroke survivors, the Stroke Impact Scale (SIS), ${ }^{10,11}$ the Stroke-Specific Quality of Life Scale, ${ }^{12}$ and the Stroke and Aphasia QOL Scale, ${ }^{13}$ that are more sensitive to short-term changes in the health status of stroke survivors than generic QOL tools are.

The SIS 2.0 was developed in the United States and first published in $1999^{10}$ as a 64-item multidimensional instrument to measure QOL in stroke survivors. The instrument has no theoretical underpinning, but the development process followed a rigorous qualitative and quantitative procedure where patients, informal caregivers, and experts in stroke were involved. ${ }^{14}$ Developers conducted several focus groups and expert panels to identify impairments, disabilities, and handicaps determined by stroke. After this process, the following 8 dimensions of the impact of stroke were identified: Strength, Hand function, Activities of daily living (ADL)/Instrumental activities of daily living (IADL), Mobility, Physical functioning, Memory, Communication, Emotion, and Social participation. In addition, the SIS 2.0 included a $100-\mathrm{mm}$ visual analog scale to measure global recovery after stroke with a separate score. The above 8-dimension structure of the SIS 2.0 was never empirically tested. Duncan et al ${ }^{10}$ reported an exploratory factory analysis where 5 factors were found, which evidently do not correspond to the 8 dimensions. Initially, the tool was tested for contrasting group validity (being able to discriminate groups of stroke survivors with different level of disability) and concurrent validity. ${ }^{10}$ Internal consistency reliability was adequate (ranging between 0.83 and 0.90 ), but test-retest reliability for the Emotional dimension was only 0.57 . The authors commented that, probably, the low reliability was a result of an item with bad item-domain correlation and to a "random variability" of all items included in this domain. In the same study analysis, the SIS 2.0 showed a floor effect in the Hand function domain $(40.2 \%$ of the sample who scored 0$)$ and a ceiling effect in the Communication domain $35.4 \%$ of the sample who scored 100) and the Social participation domain $(15.6 \%$ of the sample who scored 100$)$.

Edwards and O'Connell ${ }^{15}$ tested the SIS 2.0 in Australia. Validity was tested by item-domain correlations, which were all greater than 0.40 , and correlating the SIS 2.0 with the Zung Self-rating Depression Scale and the WHOQOL-Brief Scale. The correlations with the first scale, with the exception of the Strength domain, were all significant, with coefficients ranging from 0.27 to 0.62 . Most of the correlations with the second scale were significant, with coefficients ranging from 0.25 to 0.69 . Reliability was tested by the Cronbach's $\alpha$, which approached 0.90 for each domain with the exception of the Emotion domain, which was 0.80 . The factorial structure of the SIS 2.0 was not tested in this study.

In 2003, the SIS 2.0 evolved into the SIS 3.0 version. ${ }^{11}$ Rasch analysis was used to test the unidimensionality of each separate domain and to identify misfitting items, defined as those with an infit mean square value above 1.3. This analysis identified 5 items with an infit mean square value of 1.5 , and thus, the initial 64 items were reduced to 59. However, the whole multidimensional construct of the scale was not examined in this study because Rasch analysis can test only unidimensional construct. Reliability was not tested in this study.

Carod-Artal et $\mathrm{al}^{16}$ tested the psychometric properties of the SIS 3.0 in Brazil. Concurrent validity was examined using other scales measuring generic QOL, neurological and physical functioning, cognition, anxiety, and depression. Most of these correlations were statistically significant and ranged between 0.17 and 0.85 . Internal consistency and test-retest reliability were satisfactory for 7 domains $(0.81-0.94$ and $0.79-0.94$, respectively), whereas for the Emotional domain, internal consistency was 0.49 and test-retest reliability was 0.48 . These investigators also tested the floor and ceiling effects and found a floor effect in the Hand function dimension $(45.9 \%$ of sample that scored 0$)$ and a ceiling effect in the Communication dimension (17.3\% that scored 100). The factorial structure of the SIS 3.0 was not tested in this study.

Geyh et $\mathrm{al}^{17}$ tested the psychometric properties of the SIS 2.0 in the German population. They also used Rasch analysis to test the unidimensionality of each individual domain and found 7 items showing lack of fit to the data (with infit mean square values $>1.3$ ) in their dimensions. The person reliability index, an alternative statistics for Cronbach's $\alpha$, ranged between 0.67 (for the Communication domain) and 0.92. A floor effect was shown in the Hand function domain $(27.8 \%$ of patients who scored 0$)$ and a ceiling effect was shown in the Memory, Communication, and Hand function domains $(40.5 \%, 54.6 \%$, and $19.6 \%$ of the patients who scored 100, respectively). Other kinds of validity were not tested in the study of Geyh et al, and the authors concluded that the dimensionality of the SIS 3.0 should have been further studied.

Even though several studies have tested the psychometric properties of the SIS 2.0 and 3.0, its factorial structure has not been tested to date. This is a significant limitation to understanding the psychometric properties of the SIS 3.0. Knowing the factorial structure of a scale is considered an essential and preliminary step for its 
construct validity and reliability testing. ${ }^{18}$ In addition, the floor and ceiling effect and the reliability (internal consistency and test-retest reliability) of the SIS 3.0 have been not supported in several studies. Therefore, the aim of this study was to test the psychometric properties of the SIS 3.0, such as its construct (including the factorial structure, concurrent, and contrasting group validity), floor and ceiling effect, and reliability (internal consistency and test-retest).

\section{Methods}

\section{Design, Sample, and Setting}

A cross-sectional design was used to conduct the study. Participants enrolled in the study were all stroke survivors admitted to 16 rehabilitation facilities in the provinces of Rome, Frosinone, Foggia, Lecce, Bologna, Grosseto, Caserta, Naples, Siena, and Messina. These provinces are located in the north, center, and south of Italy. All enrolled participants had a stroke diagnosed clinically and then confirmed by tomography or magnetic resonance. Individuals were excluded from the study if they had (1) a severe preexisting neurological deficit caused by a previous neurological disorder, (2) concomitant severe systemic illness such as cancer or severe organ failure, and (3) inability to answer the questionnaires because of clinical dementia or global aphasia.

\section{Instruments}

The following instruments were used.

1. An investigator-developed sociodemographic questionnaire was used to gather general data such as gender, age, education, marital status, and employment. This is not a psychometric tool but was used in a previous study. ${ }^{19}$ Clinical data such as type (eg, ischemic) and site of stroke, and time from stroke as well, were abstracted from patients' clinical charts.

2. The SIS 3.0. ${ }^{11}$ As described above, this 59-item instrument measures QOL of stroke survivors in 8 dimensions. All items use a 5-point Likert scale, and a standardized score from 0 to 100 is calculated from each dimension, with higher scores meaning better QOL. The 100-mm visual analog scale item measures the global perception of recovery from stroke. Before its use in the Italian sample, the SIS 3.0 was translated from English into Italian by 2 nurses with expertise in stroke and fluent in English. Then, the Italian version was back-translated into English by a bilingual English teacher with expertise in medical English blinded to the original version. Then, the 2 versions were compared and minimal modifications were done. The final version was called SIS 3.0 Italian version.

3. The SF- $36^{6}$ is a generic instrument used worldwide to measure QOL. The SF-36 includes 36 items grouped in 8 scales: Physical function, Role physical,
Bodily pain, General health, Vitality, Social function, Role emotional, and Mental health. The 8 scales can be also grouped into either the Physical Component Summary (PCS) or the Mental Component Summary (MCS). Each scale is standardized to 100; higher scores mean better QOL. The SF-36 has been tested for reliability and validity in stroke survivors. ${ }^{6,20}$ Validity was tested with factor analysis and contrasting patients with different gravity conditions. Reliability was tested evaluating the internal consistency of each scale. ${ }^{6,20}$ Cronbach's $\alpha$ ranged from .68 to .98 in the present study.

4. The Barthel Index (BI), ${ }^{21}$ with 10 items, assesses independence in physical activities such as mobility, feeding, bathing, toilet using, grooming, transfer, dressing, climbing stairs, bowel, and bladder control. The possible score ranges from 0 to 100 , and a higher score means greater independence. The psychometric properties of the BI were established for validity, correlating BI scores with those of the National Institute of Health Stroke Scale (NIHSS), ${ }^{22}$ and for interrater reliability. ${ }^{22}$ In the present study, the Cronbach's $\alpha$ coefficient was .92.

5. The Mini-Mental State Examination (MMSE) ${ }^{23}$ is a 19-item measure of cognitive functions: orientation, registration, attention and calculation, recall, language, repetition, and execution of complex commands. Possible score varies from 0 to 30, and higher scores mean better cognition. The validity and reliability of the MMSE have been proven also for the stroke population. ${ }^{24}$ Specifically, concurrent validity of the MMSE was evaluated with a battery of neuropsycological tests for cognitive impairment. ${ }^{24}$ Reliability tested in this study resulted in a Cronbach's $\alpha$ of .83 .

6. The Hospital Anxiety and Depression Scale $(\mathrm{HADS})^{25}$ has been used frequently in the stroke patient population $^{26,27}$ and consists of 2 scales with 7 items each that measure anxiety and depression. Possible scores for the 2 scales range from 0 to 21, with higher scores corresponding to higher levels of anxiety and depression. The concurrent validity of the HADS in the stroke population has also been proven recently with other scales measuring anxiety and depression. ${ }^{28}$ The internal consistency reliability of the HADS in this research was adequate (Cronbach's $\alpha$ of .77 and .70 for anxiety and depression, respectively).

7. The NIHSS ${ }^{29}$ is a 15 -item instrument that assesses specific neurological functions (level of consciousness, questions, commands, gaze, visual field, facial palsy, motor leg and arm, ataxia, sensory, language, dysarthria, extinction, and inattention). Each item is summed, and the total score ranges from 0 to 42 . A higher score denotes stronger impairment due to stroke. The NIHSS has been widely used in clinical trials. ${ }^{30}$ The validity of the NIHSS was evaluated by correlating its score with brain damage size as measured by computed tomography. ${ }^{30}$ Interrater reliability was also established. ${ }^{30}$ 
8. The Modified Rankin Scale (MRS) ${ }^{31}$ is a strokespecific instrument that evaluates functional dependence. Possible scores range from 0 to 5 . Higher scores indicate more symptoms and more severe disability: at score 0 , the patient has no stroke symptoms and no disability, and at score 5 , the patient has serious disability. Concurrent validity of the MRS was tested with the BI and the NIHSS, whereas reliability was established with interobserver agreement. ${ }^{31,32}$

9. The IADL scale ${ }^{33}$ is an 8 -item tool used worldwide to assess patients' ability to perform common activities such as using a telephone, shopping, preparing food, housekeeping, doing the laundry, using transportation, managing one's own medications, and handling finances. The psychometric properties on the IADL scale have been retested also recently in the elderly population $^{34}$; criterion validity was evaluated with measures of mobility performance, and reliability, with test-retest. ${ }^{34}$ The score of the IADL scale ranges from 0 to 8 , with a higher score denoting greater ability to carry out the activities.

The institutional review boards of each rehabilitation facilities where patients were enrolled approved the study before data collection began. Patients were approached during hospitalization by research assistants trained in data collection. After patients were fully informed of the study and signed the informed consent form, an initial set of the study instruments were administered. Patients who were able and willing to complete the instruments independently did so, but most asked the research assistants, who were nurses, to have the items read. If research assistants noticed that patients were burdened by data collection, they stopped and resumed data collection in a second time (in any case within 24 hours). No more than 1 interruption on data collection was performed by research assistants. All the above instruments had a Gunning's Fog Index equals 2, which means participants needed to have 2 years of formal education to understand the instruments' content. The MMSE, the NIHSS, and the MRS were always administered by trained nurses. Fifteen days after the initial data collection, SIS 3.0 was readministered to assess test-retest reliability.

\section{Statistical Analysis}

Descriptive statistical procedures (mean, SD, frequencies) were used to describe the sociodemographic characteristic of the sample, including clinical characteristics of stroke and the mean of each of the scales' scores.

The construct validity of the SIS 3.0 was evaluated exploring its factorial structure and with concurrent and contrasting group validity. The factorial structure was evaluated by confirmatory factor analysis (CFA). Using a multifaceted approach to assess the model fit ${ }^{35}$ and taking into account the recommendations of $\mathrm{Hu}$ and
Bentler, ${ }^{36,37}$ the following fit indices were considered: $\chi^{2}$, comparative fit index (CFI), root-mean-square error of approximation (RMSEA), and standardized rootmean-square residual (SRMR). Overall model fit was judged using the following cutoff values: CFI 0.95 or greater, ${ }^{37}$ RMSEA up to 0.05 and in the lower bound of the $90 \%$ confidence interval (CI), ${ }^{38}$ and SRMR values below $0.08^{36,37}$ as indicating a good fit. Given the small sample size for the number of SIS 3.0 items (subject variable ratio $=6.6: 1$ ), the CFA was performed via item parcelling. Item parcelling is a measurement practice commonly used with latent-variable analysis techniques (eg, CFA) where 2 or more items are averaged or summed to make an aggregate-level indicator, named parcel. In this way, each parcel represents more than 1 item and all parcels are a reduced form of the original scale. This approach allows the implementation of analytical techniques even with small sample size for the number of the instrument items. ${ }^{39,40}$ Because unidimensionality is a prerequisite for item parceling, we verified parcel unidimensionality through exploratory factor analysis (EFA) and then reported Cronbach's $\alpha$ for each parcel. ${ }^{39,40}$

The concurrent validity of the SIS 3.0 was evaluated correlating SIS 3.0 summed score dimensions with scores of other instruments, specifically SF-36, BI, MMSE, HADS, NIHSS, and IADL, using Pearson productmoment correlations. To control for capitalization on chance due to multiple correlations, a Bonferroni-like approach was used; accordingly, only differences associated to a less than .003 probability level were considered as statistically significant. Contrasting group validity was assessed by analysis of variance comparing SIS 3.0 scores by the 6 groups of patients identified by the MRS.

Floor and ceiling effects were determined by computing the percentage of the patients who obtained the minimum and the maximum score in a measure, respectively. An instrument is considered not having a floor and a ceiling effect if both percentages are less than $15 \%{ }^{41}$

Internal consistency and test-retest reliability of SIS 3.0 were computed by Cronbach's $\alpha$ and intraclass correlation coefficient (ICC), respectively, on the dimensions extracted by factor analysis.

Data analysis was performed with SPSS 19.0 and Mplus 6.12. Level of significance was set at $P<.05$.

\section{Results}

\section{Patients' Sociodemographic and Clinical Characteristics}

A total of 450 patients were initially approached, but 58 were excluded because of exclusion criteria $(n=40)$ or refusal to participate in the study $(\mathrm{n}=18)$. The final sample consisted of 392 stroke survivors, whose 


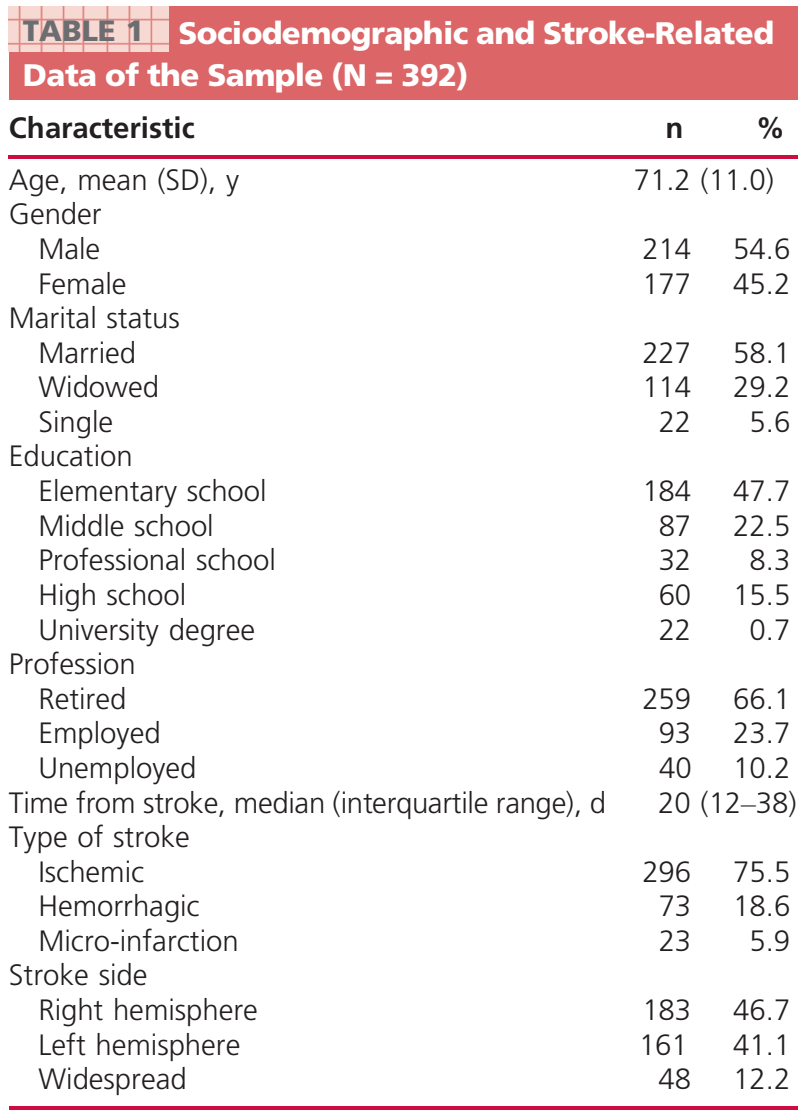

sociodemographic and stroke-related characteristics are reported in Table 1. The mean (SD) age of participants was 71 (11) years; most were men $(55 \%)$ and married $(58 \%)$. Educational level was low, with $70 \%$ of the sample educated at only an elementary or middle school level. Retired people constituted $66 \%$ of the sample. Twenty days was the median time since stroke event and $75 \%$ of the sample had had an ischemic stroke, with a slight higher prevalence of the right hemisphere $(47 \%)$.

Table 2 reports the scores on each of the instruments. In this sample, the mean scores on the NIHSS and MRS were 7.2 and 3.2, respectively, indicative of slight to moderate neurological dysfunction and needing help even though capable to walk. The mean scores on the BI and IADL scale were 44 and 3.5, respectively, which denote a need for help in activity and IADL. The mean (SD) MMSE and anxiety and depression scores in the HADS were 2 (8), 10 (5), and 11 (4), respectively, which are considered moderate cognitive impairment, moderate anxiety, and depression. Of the 8 SF-36 scales' scores, 5 were below 50 points as well as the PCS and the MCS, indicating diminished QOL in each domain. Table 2 also reports normative data collected on a random sample of 258 healthy patients aged 65 to 74 years. ${ }^{42}$ The SD of the SF-36 Role Physical and SF-36 Role Emotional were higher than their mean scores, denoting a high variability in these 2 measures. However, a similar phenomenon can been seen also in the normative data, which report a large SD in the above scales.

\section{Psychometric Evaluation of the Stroke Impact Scale 3.0}

\section{Construct Validity}

The CFA performed on the 8-factor structure of the SIS 3.0 did not fit the data $\left(\chi_{1624}^{2}=6449.9 ; P<.001\right.$; CFI, 0.82; RMSEA, 0.09 [90\% CI, 0.08-0.09]; SRMR, 0.08) and showed extremely high factor correlations among some domains $(r=0.85$ between Communication and

\section{TABLE 2 Scale Scores in Stroke Survivors}

\begin{tabular}{lccc}
\hline Scales & Mean (SD) & Potential Score Range & Italian Normative Data, ${ }^{a}$ Mean (SD) \\
\hline NIHSS & $7.2(6.3)$ & $0-42$ & - \\
MRS & $3.2(1.2)$ & $0-5$ & - \\
BI & $44.0(28.3)$ & $0-100$ & - \\
IADL & $3.5(2.7)$ & $1-8$ & - \\
MMSE & $20.2(7.9)$ & $0-30$ & - \\
HADS-A & $9.7(4.7)$ & $0-21$ & $67.3(26.0)$ \\
HADS-D & $10.9(4.4)$ & $0-21$ & $60.0(40.4)$ \\
SF-36 & & & $62.8(29.1)$ \\
Physical Function & $20.9(26.6)$ & $0-100$ & $51.6(21.5)$ \\
Role Physical & $9.7(25.9)$ & $0-100$ & $55.0(21.1)$ \\
Bodily Pain & $47.7(34.7)$ & $0-100$ & $72.9(24.9)$ \\
General Health & $52.1(20.8)$ & $0-100$ & $70.50(36.80)$ \\
Vitality & $50.8(20.7)$ & $0-100$ & $60.4(21.40)$ \\
Social Function & $46.2(23.1)$ & $0-100$ & -100 \\
Role Emotional & $14.60(29.30)$ & $0-100$ & \\
Mental Health & $55.60(19.10)$ & &
\end{tabular}

Abbreviations: BI, Barthel Index; HADS-A, Hospital Anxiety and Depression Scale-Anxiety Scale; HADS-D, Hospital Anxiety and Depression

Scale-Depression Scale; IADL, Instrumental Activity of Daily Living; MMSE, Mini-Mental State Examination; NIHSS, National Institute of Health Stroke Scale; MRS, Modified Rankin Scale; SF-36, 36-item Short-Form Health Survey.

The Italian normative data have been taken from a random healthy population aged $65-74$ years $(n=258)$. 
Memory; $r=0.93$ between ADL/IADL and Mobility; $r=0.86$ between ADL/IADL and Hand function). To examine SIS 3.0 factor structure, we first performed EFA to identify the number of factors and the items loading per each factor, then we validated the resulting factor solution using CFA.

Before EFA, the Bartlett test of sphericity and Kaiser-Meyer-Olkin test were performed to support the use of EFA for the data. The Bartlett test of sphericity resulted in the following values: $\chi_{1711}^{2}=27433.9, P<$ .001 ; the Kaiser-Meyer-Olkin test was 0.96. Both tests indicated sample adequacy for conducting EFA. In the EFA, in deciding among the various factor solutions, a scree plot of eigenvalues was considered along with the interpretability of the solution. A solution is considered to be interpretable if items load on a factor (pattern coefficient $\geq 0.30$ ) and appear to align in content. To choose the best solution, the following 4 criteria were considered: (1) the number of items not loading on any factor, (2) the presence of factors with few items, (3) the number of items loading on more than 1 factor, and (4) the interpretability of the factors. ${ }^{43}$ Factors were extracted using principal axis factoring and then oblique rotated using the Promax procedure.

In the EFA, the analysis of the eigenvalues (Figure 1) suggested 3 plausible solutions: 8 factors, 6 factors, and 4 factors. Because both the 8 - and 6-factor solutions were not interpretable or simple (particularly, the 8-factor solution did not reproduce the 8 original dimensions), a 4-factor solution was preferred (Table 3 ). Moreover, this solution was the most favorable with respect to all the 4 criteria chosen for conducting the EFA. ${ }^{43}$ The first factor that resulted from the EFA, labeled "Physical," explained $32 \%$ of the total variance; the second factor, "Cognitive," explained $18 \%$ of the total variance; the third factor, "Emotional," explained $7 \%$ of the total variance; and the fourth factor, "Social participation," explained $6 \%$ of the total variance. Overall, these factors explained $63 \%$ of

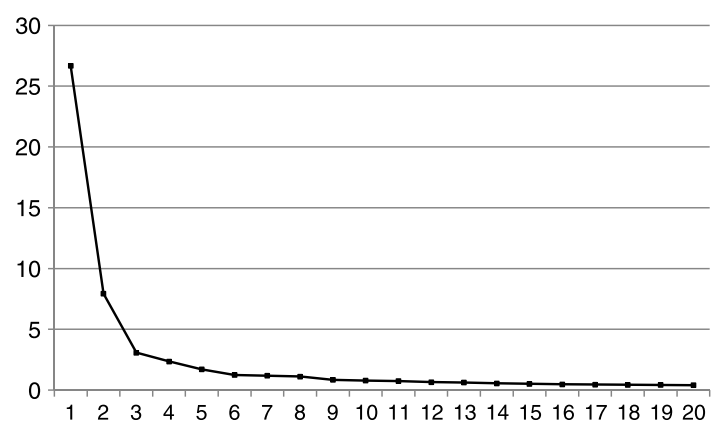

FIGURE 1. Scree plot of eigenvalues. The number of eigenvalue is 59 (as the number of items), but we have reported here only the first 20 . total variance and showed low to moderate positive correlations $^{44}$ (Table 4).

As reported above, before testing the new factorial structure of the SIS 3.0 with CFA, the SIS 3.0 items underwent item parcelling (see Table 5 for parcels' details). The CFA implemented for the 4-factor model resulted from the EFA fitted the data: $\chi^{2}{ }_{90}=342.99$; $P<.001$; CFI, 0.96; RMSEA, 0.085 (90\% CI, 0.076-0.095); SRMR, 0.053. Factor loadings were all significant $(P<.01)$ and higher than 0.60 . Correlations among factors ranged from 0.38 (between the Physical and Emotional factors) to 0.76 (between the Social participation and Physical factors) (Figure 2).

The concurrent validity of the new 4 SIS 3.0 factors (Table 6) showed weak to moderate correlations with all SF-36 scales, PCS, and MCS. Correlations with the other scales were almost all significant, ranging from -0.27 to 0.69 . The highest correlations were between the SIS 3.0 Cognitive factor and the MMSE $(r=0.69)$, between the SIS 3.0 Physical factor and the BI and IADL scale ( $r=0.69$ for both scales), and between the SIS 3.0 Emotional factor and the HADS-Anxiety and HADS-Depression ( $r=-0.68$ and -0.67 , respectively).

Contrasting group validity was also supported. Using the analysis of variance test, the comparison of the SIS 3.0 factor scores among the 5 groups of patients identified by the MRS was significant $(P<0.001)$ : the groups of patients with lower MRS had higher SIS 3.0 factor scores and vice versa (Table 7).

\section{Floor and Ceiling Effect and Reliability}

The lowest floor effect was observed for the Cognitive and Emotional factors (Table 8 ), where only $0.5 \%$ of patients scored 0 . The highest floor effect was observed in the Social participation domain (7.1\%). The Cognitive factor was also the factor with the highest ceiling effect, with $4.3 \%$ of participants who scored 100 .

Internal consistency reliability (Cronbach's $\alpha$ ) was high in all SIS 3.0 factors, ranging from 0.89 for the Emotional factor to 0.98 for the Physical factor. The test-retest reliability showed high ICCs, which ranged from 0.79 for the SIS 3.0 Stroke global recovery item to 0.93 for the Cognitive factor (Table 8 ).

\section{Discussion}

To our knowledge, this is the first study that has tested the factorial structure of the SIS 3.0 using CFA. Our analysis adds new insight into the instrument's psychometric properties. Surprisingly, the 8-dimension solution did not fit the data well using CFA, so it was necessary to explore the factorial structure using EFA and then CFA. This analysis resulted in a 4-factor solution that fit the data well with supportive fit indices. 
Factor 2:

Factor 3:

Factor 3: Social

In the past week, how would you rate the strength of your...

Physical

(1a) Arm that was most affected by your stroke?

(1b) Grip of your hand that was most affected by your stroke?

(1c) Leg that was most affected by your stroke?

0.81

0.86

0.75

(1d) Foot/ankle that was most affected by your stroke?

In the past 2 weeks, how difficult was it to...

(5a) Cut your food with a knife and fork?

(5b) Dress the top part of your body?

(5c) Bathe yourself?

(5d) Clip your toenails?

(5e) Get to the toilet on time?

(5f) Control your bladder (not have an accident)?

(5h) Do light household tasks/chores (eg, dust, make a bed, take out garbage, do the dishes)?

(5i) Go shopping?

(5j) Do heavy household chores (eg, vacuum, laundry or yard work)?

In the past 2 weeks, how difficult was it to...

(6a) Stay sitting without losing your balance?

(6b) Stay standing without losing your balance?

(6c) Walk without losing your balance?

(6d) Move from a bed to a chair?

(6e) Walk one block?

(6f) Walk fast?

(6g) Climb one flight of stairs?

(6h) Climb several flights of stairs?

(6i) Get in and out of a car?

In the past 2 weeks, how difficult was it to use your hand that was most affected by your stroke to..

(7a) Carry heavy objects (eg, bag of groceries)?

(7b) Turn a doorknob?

(7c) Open a can or jar?

(7d) Tie a shoelace?

(7e) Pick up a dime?

In the past week, how difficult was it for you to...

(2a) Remember things that people just told you?

(2b) Remember things that happened yesterday?

(2c) Remember to do things (eg, keep scheduled appointments or take medication)?

(2d) Remember the day of the week?

(2f) Concentrate?

(2g) Think quickly?

(2h) Solve problems?

In the past week, how difficult was it to...

(4a) Say the name of someone whose face was in front of you?

(4b) Understand what was being said to you in a conversation?

(4c) Reply to questions?

(4d) Correctly name objects?

(4e) Participate in a conversation with a group of people?

(4f) Have a conversation on the telephone?

(4g) Call another person on the telephone (select the correct phone number and dial)?

In the past 2 weeks, how difficult was it to..

(5g) Control your bowels (not have an accident)?

In the past week, how often did you...
(3a) Feel sad?
(3b) Feel that there is nobody you are close to?
(3c) Feel that you are a burden to others?
(3d) Feel that you have nothing to look forward to?
(3e) Blame yourself for mistakes?
(3f) Enjoy things as much as you ever have?
(3g) Feel quite nervous?
(3h) Feel that life is worth living?

0.77

\subsection{4}

0.88

0.90

0.82

0.87

0.46

0.83

0.80

0.65

0.69

0.90

0.91

0.91

0.84

0.80

0.83

0.78

0.82

0.81

0.81

0.81

0.80

0.79

$-0.04$

$-0.08$

0.04

$-0.05$

$-0.15$

$-0.06$

0.15

$-0.03$

$-0.09$

0.04

0.01

$-0.01$

0.11

0.26

0.42

0.00

$-0.15$

0.04

$-0.01$

0.09

0.06

0.00

0.00 Cognitive

Emotional

Participation

$$
\begin{array}{r}
0.03 \\
-0.02 \\
0.01
\end{array}
$$

0.00

0.08

0.10

0.03

$-0.05$

0.00

0.38

$-0.02$

0.00

$-0.09$

0.26

0.02

$-0.05$

0.02

$-0.03$

$-0.08$

$-0.09$

$-0.10$

$-0.08$

$-0.04$

0.06

0.03

$-0.02$

$-0.01$

0.83

0.90

0.87

0.93

0.84

0.84

0.66

0.80

0.80

0.83

0.80

0.83

0.77

0.69

0.46

$-0.06$

0.27

0.04

0.06

0.06

$-0.10$

$-0.13$

0.11
0.18

0.17

0.23

0.20

$-0.01$

0.00

$-0.02$

$-0.01$

$-0.05$

$-0.11$

$-0.11$

$-0.10$

$-0.02$

0.01

$-0.03$

$-0.06$

$-0.03$

$-0.03$

$-0.03$

$-0.01$

0.00

$-0.01$

$-0.02$

$-0.02$

$-0.01$

$-0.01$

$-0.04$

$-0.03$

$-0.07$

$-0.05$

$-0.13$

0.01

$-0.04$

$-0.05$

0.08

0.11

0.13

0.11

0.05

0.06

$-0.04$

$-0.12$

0.71

0.63

0.67

0.78

0.75

0.49

0.65

0.47
$-0.01$

$-0.23$

$-0.26$

$-0.15$

$-0.15$

$-0.09$

$-0.07$

$-0.01$

0.08

0.03

$-0.01$

0.12

0.18

0.28

$-0.14$

$-0.04$

0.02

$-0.08$

0.09

0.21

0.14

0.20

0.16

0.07

$-0.02$

0.01

0.07

0.06

0.09

0.10

0.01

0.07

0.22

0.20

0.21

$-0.11$

0.03

$-0.16$

$-0.18$

$-0.01$

$-0.05$

0.01

0.18

$-0.04$

0.14

0.08

$-0.10$

0.24

0.11

$-0.03$

(continues) 
TABLE 3 Exploratory Factor Analysis of the Stroke Impact Scale 3.0 With Oblique Rotation, Continued

\begin{tabular}{|c|c|c|c|c|}
\hline SIS 3.0 Items & $\begin{array}{l}\text { Factor 1: } \\
\text { Physical }\end{array}$ & $\begin{array}{l}\text { Factor 2: } \\
\text { Cognitive }\end{array}$ & $\begin{array}{l}\text { Factor 3: } \\
\text { Emotional }\end{array}$ & $\begin{array}{c}\text { Factor 3: Social } \\
\text { Participation }\end{array}$ \\
\hline (3i) Smile and laugh at least once a day? & -0.08 & 0.05 & 0.59 & 0.11 \\
\hline \multicolumn{5}{|l|}{$\begin{array}{l}\text { During the past } 4 \text { weeks, how much of the time have you been } \\
\text { limited in... }\end{array}$} \\
\hline (8a) Your work, volunteer or other activities? & 0.36 & -0.15 & 0.01 & 0.51 \\
\hline (8b) Your social activities? & 0.13 & 0.05 & 0.10 & 0.64 \\
\hline (8c) Quiet recreation (crafts, reading)? & 0.15 & 0.13 & 0.00 & 0.62 \\
\hline (8d) Active recreation (sports, outings, travel)? & 0.32 & -0.13 & 0.11 & 0.56 \\
\hline (8e) Your role as a family member and/or friend? & -0.07 & 0.20 & 0.10 & 0.65 \\
\hline (8f) Your participation in spiritual or religious activities? & 0.05 & 0.20 & 0.03 & 0.52 \\
\hline (8h) Your ability to control your life as you wish? & 0.03 & 0.01 & 0.12 & 0.73 \\
\hline (8i) Your ability to help others in need? & 0.08 & 0.01 & 0.06 & 0.74 \\
\hline
\end{tabular}

Boldface identifies the primary factor on which the item loaded.

These results are somewhat consistent with results of other researchers. In our analysis, 4 of the dimensions proposed by Duncan et al ${ }^{10,11}$ (Strength, Hand function, Mobility, ADL/IADL) converged in a unique factor. Indeed, these 4 dimensions were found highly correlated in our initial CFA and clustered in a unique factor (which we called Physical). Therefore, using these dimensions individually as proposed by instrument developers in clinical practice would not provide any more specific information regarding the impact of stroke. In fact, in previous studies in which Strength, Hand function, Mobility, and ADL/IADL dimensions were tested for their concurrent validity, ${ }^{16,19,45}$ similar correlation coefficients were found among the above 4 dimensions and concurrent validity scales. Also, in several intervention studies where the SIS scores were the outcome variables, the above 4 dimensions measured individually behaved very similarly. ${ }^{46-49}$

In this study, the items of the original SIS 3.0 Memory and Communication dimensions clustered together in a unique factor that we called Cognitive factor. Indeed, the Memory and Communication domains were highly correlated in our initial CFA $(r=$ $0.85)$, providing evidence of very similar item contents between the 2 domains. Similarly, in the Australian validity and reliability testing of the SIS 2.0 , researchers found a correlation of $r=0.70$ between the Memory and Communication dimensions. ${ }^{15}$ In addition, in several SIS validity and intervention studies, ${ }^{16,19,45}$ the Memory and Communication domains correlated with similar coefficients with other concurrent validity scales and behaved similarly after rehabilitation interventions. ${ }^{46-50}$ In the new factorial structure of the SIS 3.0 provided in this study, the 4 factors (Physical, Cognitive, Emotional, and Social participation) have only a moderate correlation with each other, which is evidence that they measure distinct domains. In the EFA, item $5 \mathrm{~g}$, which investigates how often in the last week the patient found it difficult to control the bowels, cross-loaded between the Physical and the Cognitive factors. This result was not a surprise because people with cognitive impairment are also at higher risk of fecal incontinence. ${ }^{51,52}$

Most correlations between SIS 3.0 factors and SF-36 subscales were significant. Interestingly, the SIS 3.0 Social participation factor showed a stronger correlation with the SF-36 Physical Functioning than with the SF-36 Social Functioning domain. Actually, the items of the SIS 3.0 Social participation factor assess limitations in 8 specific activities (eg, work, active recreation) that require physical abilities that may be compromised in stroke survivors, whereas the 2 items on the SF-36 Physical Functioning generically measure how physical health or emotional problems interfere with "normal social activities."

Correlations between SIS 3.0 factors and the other concurrent validity scales were all highly significant and congruent in their correlations (eg, the Physical factor showed the strongest correlation with the BI, and the Cognitive factor, with the MMSE). These results are consistent with other studies that tested the concurrent validity of the SIS 3.0. ${ }^{10,16}$

Contrasting group validity testing showed mean score differences among the MRS categories: as the condition of stroke survivors worsened, SIS 3.0 factor scores significantly decreased $(P \leq .001)$. These results demonstrate that the new SIS 3.0 factors are able to discriminate across different degrees of stroke severity and might thus be useful in clinical practice and research

\begin{tabular}{|c|c|c|c|c|}
\hline \multicolumn{5}{|c|}{$\begin{array}{l}\text { Among Factors Identified by Exploratory } \\
\text { Factor Analysis }\end{array}$} \\
\hline Factors & 1 & 2 & 3 & 4 \\
\hline 1. Physical & & & & \\
\hline 2. Cognitive & 0.44 & & & \\
\hline 3. Emotional & 0.34 & 0.53 & & \\
\hline 4. Social Participation & 0.60 & 0.36 & 0.31 & 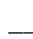 \\
\hline
\end{tabular}

All correlations are significant for $P<.01$. 
TABLE 5 Item Parceling of the Stroke Impact Scale 3.0

\begin{tabular}{|c|c|c|c|}
\hline Factor & Parcel & $\begin{array}{l}\text { Items Included } \\
\text { in Parcel }\end{array}$ & $\begin{array}{l}\text { Cronbach's } \alpha \\
\text { of the Parcel }\end{array}$ \\
\hline \multirow[t]{7}{*}{ Physical } & P1 & $1 a, 1 b, 1 c, 1 d$ & .93 \\
\hline & P2 & $5 a, 5 b, 5 c, 5 d$ & .94 \\
\hline & P3 & $5 e, 5 f, 5 g$ & .85 \\
\hline & P4 & $5 h, 5 i, 5 l$ & .93 \\
\hline & P5 & $6 \mathrm{a}, 6 \mathrm{~b}, 6 \mathrm{c}, 6 \mathrm{~d}$ & .94 \\
\hline & P6 & $6 f, 6 g, 6 h, 6 i, 6 j$ & .97 \\
\hline & P7 & $7 a, 7 b, 7 c, 7 d, 7 e$ & .96 \\
\hline \multirow[t]{4}{*}{ Cognitive } & P1 & $2 a, 2 b, 2 c, 2 d$ & .94 \\
\hline & P2 & $2 f, 2 g, 2 h$ & .92 \\
\hline & P3 & $4 a, 4 b, 4 c, 4 d$ & .92 \\
\hline & P4 & $4 e, 4 f, 4 g$ & .91 \\
\hline \multirow[t]{3}{*}{ Emotional } & P1 & $3 a, 3 b, 3 c$ & .77 \\
\hline & P2 & $3 d, 3 e, 3 g$ & .79 \\
\hline & P3 & $3 f, 3 h, 3 i$ & .72 \\
\hline \multirow{2}{*}{$\begin{array}{l}\text { Social } \\
\text { participation }\end{array}$} & P1 & $8 a, 8 b, 8 c, 8 d$ & .87 \\
\hline & P2 & $8 \mathrm{e}, 8 \mathrm{f}, 8 \mathrm{~h}, 8 \mathrm{i}$ & .87 \\
\hline
\end{tabular}

to monitor stroke progression and rehabilitation outcomes.

Previous studies on the SIS 3.0 psychometric characteristics $^{10,16,17}$ have shown poor internal consistency and test-retest reliability in the Emotion and Communication domains (with a Cronbach's $\alpha$ and ICC $<0.70$ ), ceiling effects in the Communication and Social participation dimension, and a floor effect in the Hand function dimension (with $>15 \%$ of the patients reaching the highest and the lowest possible score in these domains, respectively). In our study, the internal consistency and test-retest reliability of the
SIS 3.0 Emotional factor were 0.88 and 0.82 , respectively. The highest floor effect was observed in the SIS 3.0 Social participation factor but was only $7.1 \%$. The highest ceiling effect $(4.3 \%)$ was exhibited by the SIS 3.0 Cognitive factors. This is an important finding because the 4 SIS 3.0 factors identified in this study showed better reliability and limited floor and ceiling effect. Compared with the study of Carod-Artal et al, ${ }^{16}$ our patients had even more limited functional independence according to the BI, the NIHSS, and the MRS scores, and thus, they should have exhibited a higher floor effect. Test-retest reliability showed also that the SIS 3.0 was very stable with the 15 -day period of administration.

\section{Limitation}

This study has several limitations. First, participants were selected from rehabilitation hospitals and had more severe limitations as is expected in a recent stroke. That is, survivors in the chronic phase of the stroke living in a community setting were not enrolled in this study. In addition, participants who had severe or preexisting neurological disorders and severe comorbidity were excluded. The selection of a more homogeneous sample of stroke survivors resulted in slightly less generalizability of findings but allowed for control of possible confounders, resulting in more confidence in the validity of the SIS 3.0 for this population. Another limitation of the study is that, sometimes, patients completed the research instruments by their own while others asked the instruments' item to be

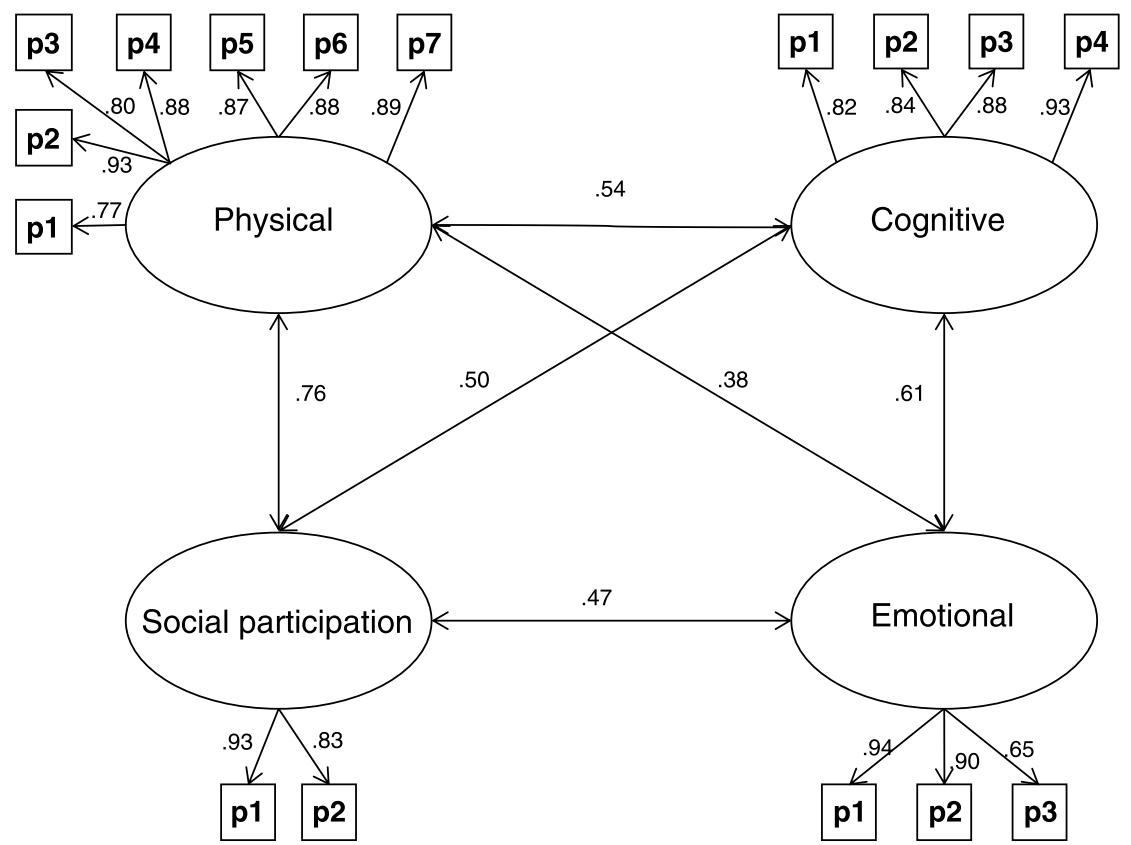

FIGURE 2. Confirmatory factor analysis of the Stroke Impact Scale 3.0. P indicates parcel. 

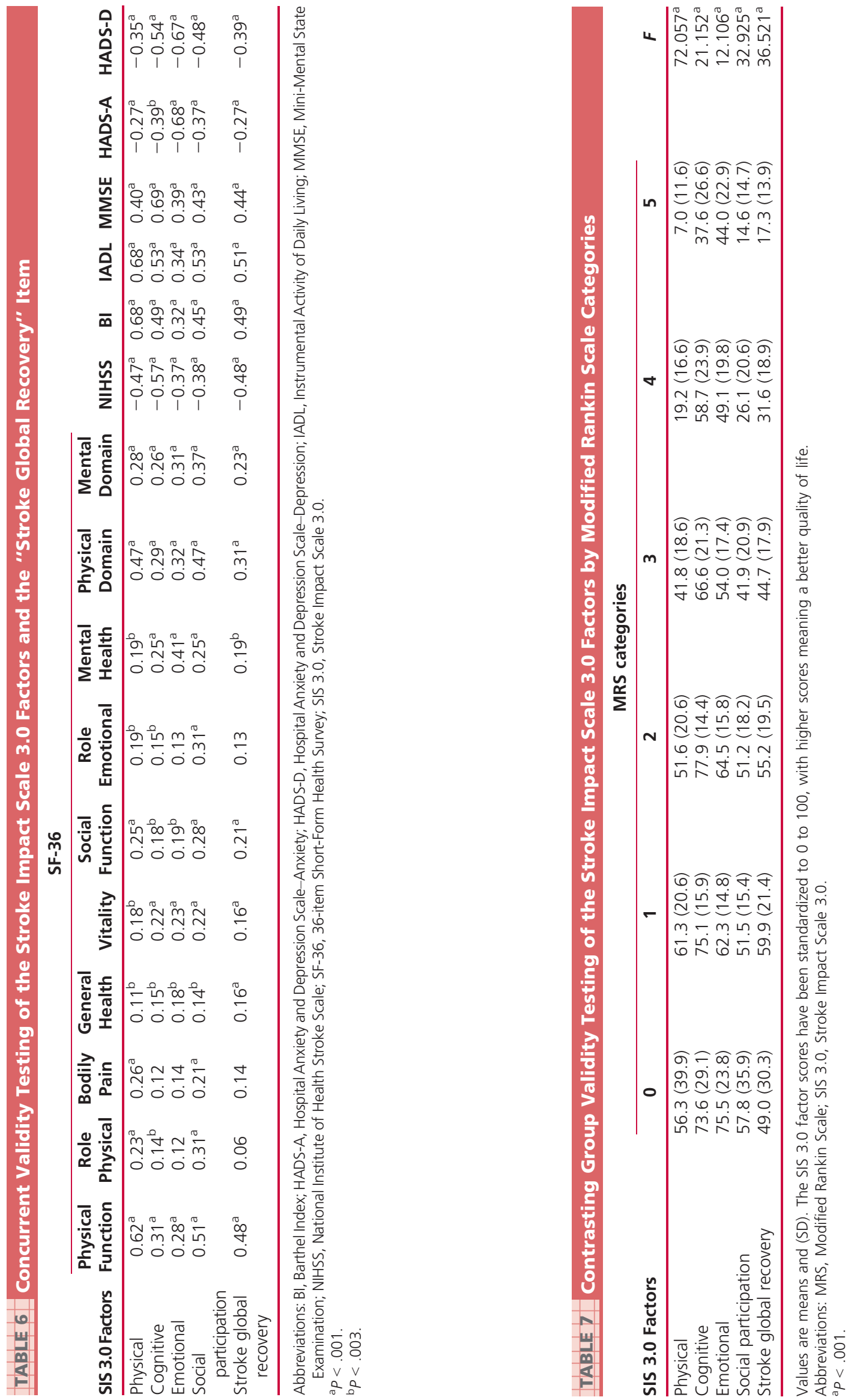


\section{What's New and Important}

Confirmatory factor analysis revealed a new 4-factor structure of the SIS 3.0 with supportive fit indices.

- The new 4-factor structure of the SIS 3.0 showed better reliability and lower floor and ceiling effect than the original 8-factor structure did.

- The use of SIS 3.0 in clinical practice and research is recommended.

read by research assistants. Even though research assistants were trained to administer the instruments without influencing patients' responses, there might be difference on scale scores depending and the way the scales were administered. However, the self-administration of research instruments might be a challenge for stroke survivors because of the physical disabilities, as other investigators have also reported. ${ }^{11,17}$ We observed that within some factors (eg, Physical and Cognitive factors), there were items highly correlated, suggesting a redundancy among items. This might represent a limitation to a faster administration of the instrument including, at the moment, 59 items. Finally, our analysis used a sample of Italian stroke survivors; thus, validating the new factorial structure in a more diverse population is indicated.

\section{Implications for Clinical Practice}

The results of our study indicate that the SIS 3.0 is an instrument that exhibits improved evidence of validity and reliability to evaluate QOL in stroke survivors undergoing rehabilitation. Compared with previous studies, ${ }^{10,16}$ the new factorial structure of the SIS 3.0 better fits the data, measures distinct dimensions, has better reliability, and has lower floor and ceiling effects. These results suggest that using the 4 factors with 4 separate scores in clinical practice would allow a better and not redundant evaluation of stroke survivors' QOL than using the 8 original domains. In fact, according to the results of our study, computing separate scores for the Strength, ADL/IADL, Mobility, and Hand function domains (which were found to be in the Physical factor) as well as for the Communication and Memory domains (which were found to be in the Cognitive factor) would not yield more specific information than computing 2 scores for the Physical and Cognitive factors, respectively. Better reliability and lower floor and ceiling effects of the 4 factors mean that computing 4 scores ( 1 per each factor) will yield a more precise evaluation of stroke survivors' QOL with less measurement error ${ }^{53}$ and with a reduced portion of people with the highest and the lowest possible scores (because of ceiling and floor effects). Consequently, stroke recovery might be better evaluated as well as interventions aimed at improving recovery. Indeed, specific instruments to measure QOL in stroke survivors such as the SIS 3.0 have been developed in the last 2 decades to be more sensitive to patients' changes and to avoid the ceiling and floor effect of prior-developed tool. ${ }^{54,55}$

Because construct validity deals with the theoretical adequacy of a measure, another clinical implication of this study is that the 4-factor structure of the SIS 3.0 gives a theoretical base to QOL in stroke survivors that can be used to guide clinical practice. In fact, the Physical, Cognitive, Emotional, and Social participation dimensions may illuminate clinicians on the focus of interventions to improve recovery and QOL after stroke. ${ }^{56}$

\section{Conclusion}

Further studies are needed to evaluate the psychometric properties of the SIS 3.0. As emphasized by Waltz et al, ${ }^{57}$ "one validates not the measurement tool or method but rather some use to which the measure is put"(p19) and "evidence for reliability and validity of a tool or method is accrued over time." ${ }^{(\mathrm{p} 20)}$ Further studies should be also focused on reducing the number of items because some were very highly correlated and thus probably reflect the same content.

TABLE 8 Floor and Ceiling Effects and Reliability of the Stroke Impact Scale 3.0 Factors

\begin{tabular}{|c|c|c|c|c|c|c|}
\hline \multirow[b]{2}{*}{ SIS 3.0 Factors } & \multirow[b]{2}{*}{ Mean (SD) } & \multirow[b]{2}{*}{ Range } & \multirow[b]{2}{*}{ Floor Effect, \% } & \multirow[b]{2}{*}{ Ceiling Effect, \% } & \multicolumn{2}{|c|}{ Reliability } \\
\hline & & & & & Cronbach's $\alpha$ & $\mathrm{ICC}^{\mathrm{a}}$ \\
\hline Physical & $32.7(25.3)$ & $0-100$ & 3.1 & 0.3 & .98 & 0.91 \\
\hline Cognitive & $63.3(24.3)$ & $0-100$ & 0.5 & 4.3 & .97 & 0.93 \\
\hline Emotional & 53.9 (19.9) & $0-100$ & 0.5 & 0.8 & .89 & 0.82 \\
\hline Social Participation & $35.6(23.3)$ & $0-100$ & 7.1 & 1.0 & .92 & 0.87 \\
\hline Stroke global recovery & $40.9(22.5)$ & $0-95$ & 3.1 & 0.0 & NA & 0.79 \\
\hline
\end{tabular}

Abbreviations: ICC, intraclass correlation coefficient; NA, not applicable; SIS 3.0, Stroke Impact Scale 3.0.

${ }^{\mathrm{a} A l l} P<.001$. 


\section{REFERENCES}

1. Norrving B, Kissela B. The global burden of stroke and need for a continuum of care. Neurology. 2013;80(3 suppl 2): S5-S12.

2. Daniel K, Wolfe CD, Busch MA, McKevitt C. What are the social consequences of stroke for working-aged adults? A systematic review. Stroke. 2009;40(6):e431-e440.

3. Nichols M, Townsend N, Luengo-Fernandez R, et al. European Cardiovascular Disease Statistics 2012. Brussels, Belgium: European Heart Network and European Society of Cardiology; 2012.

4. Ovbiagele B, Goldstein LB, Higashida RT, et al. Forecasting the future of stroke in the United States: a policy statement from the American Heart Association and American Stroke Association. Stroke. 2013;44:2361-2375.

5. Jonsson AC, Lindgren I, Hallstrom B, Norrving B, Lindgren A. Determinants of quality of life in stroke survivors and their informal caregivers. Stroke. 2005;36(4):803-808.

6. Ware JE, Sherbourne CD. The MOS 36-item Short-Form Health Survey (SF-36). Med Care. 1992;30:473-481.

7. Bergner M, Bobbitt R, Pollard W, Martin D, Gilson B. The Sickness Impact Profile: validation of a health status measure. Med Care. 1976;14:57-67.

8. Hunt S, McEwan T. The development of a subjective health indicator. Sociol Health Illn. 1980;2:231-246.

9. The WHOQOL Group. Development of the World Health Organization WHOQOL-BREF quality of life assessment. Psychol Med. 1998;28:551-558.

10. Duncan PW, Wallace D, Lai SM, Johnson D, Embretson S, Laster LJ. The Stroke Impact Scale version 2.0: evaluation of reliability, validity, and sensitivity to change. Stroke. 1999; 30(10):2131-2140.

11. Duncan PW, Bode RK, Min Lai S, Perera S. Rasch analysis of a new stroke-specific outcome scale: the Stroke Impact Scale. Arch Phys Med Rehabil. 2003;84(7):950-963.

12. Williams LS, Weinberger M, Harris LE, Clark DO, Biller J. Development of a stroke-specific quality of life scale. Stroke. 1999;30(7):1362-1369.

13. Hilari K, Lamping DL, Smith SC, Northcott S, Lamb A, Marshall J. Psychometric properties of the Stroke and Aphasia Quality of Life Scale (SAQOL-39) in a generic stroke population. Clin Rehabil. 2009;23(6):544-557.

14. Duncan PW, Lai SM, Keighley J. Defining post-stroke recovery: implications for design and interpretation of drug trials. Neuropharmacology. 2000;39(5):835-841.

15. Edwards B, O'Connell B. Internal consistency and validity of the Stroke Impact Scale 2.0 (SIS 2.0) and SIS-16 in an Australian sample. Qual Life Res. 2003;12(8):1127-1135.

16. Carod-Artal FJ, Coral LF, Trizotto DS, Moreira CM. The Stroke Impact Scale 3.0: evaluation of acceptability, reliability, and validity of the Brazilian version. Stroke. 2008;39(9): 2477-2484.

17. Geyh S, Cieza A, Stucki G. Evaluation of the German translation of the Stroke Impact Scale using Rasch analysis. Clin Neuropsychol. 2009;23(6):978-995.

18. Brown TA. Confirmatory Factor Analysis for Applied Research. New York, NY: The Guilford Press; 2006.

19. Vellone E, Savini S, Barbato N, Carovillano G, Caramia M, Alvaro R. Quality of life in stroke survivors: first results from the reliability and validity of the Italian version of the Stroke Impact Scale 3.0. Ann Ig. 2010;22(5):469-479.

20. McHorney CA, Ware JE, Raczek AE. The MOS 36-item Short-Form Health Survey (SF-36), II: psychometric and clinical tests of validity in measuring physical and mental health constructs. Med Care. 1993;31:247-263.

21. Mahoney FI, Barthel DW. Functional evaluation: the Barthel Index. Md State Med J. 1965;14:61-65.
22. Della Pietra GL, Savio K, Oddone E, Reggiani M, Monaco F, Leone MA. Validity and reliability of the Barthel index administered by telephone. Stroke. 2011;42(7):2077-2079.

23. Folstein MF, Folstein SE, McHugh PR. Mini Mental State: a practical method for grading the cognitive state of the patients for the clinicians. J Psychiatr Res. 1975;12: 189-198.

24. Cumming TB, Churilov L, Linden T, Bernhardt J. Montreal Cognitive Assessment and Mini-Mental State Examination are both valid cognitive tools in stroke. Acta Neurol Scand. 2013;128(2):122-129.

25. Zigmond AS, Snaith RP. The Hospital Anxiety and Depression Scale. Acta Psychiatr Scand. 1983;67:361-370.

26. Sagen U, Finset A, Moum T, et al. Early detection of patients at risk for anxiety, depression and apathy after stroke. Gen Hosp Psychiatry. 2010;32(1):80-85.

27. Sagen U, Vik TG, Moum T, Morland T, Finset A, Dammen T. Screening for anxiety and depression after stroke: comparison of the Hospital Anxiety and Depression Scale and the Montgomery and Asberg Depression Rating Scale. J Psychosom Res. 2009;67(4):325-332.

28. Kang HJ, Stewart R, Kim JM, et al. Comparative validity of depression assessment scales for screening poststroke depression. J Affect Disord. 2013;147(1-3):186-191.

29. Brott T, Adams HP, Olinger CP, et al. Measurements of acute cerebral infarction: a clinical examination scale. Stroke. 1989; 20:864-870.

30. Appelros P, Terent A. Characteristics of the National Institutes of Health Stroke Scale: results from a population-based stroke cohort at baseline and after one year. Cerebrovasc Dis. 2004;17:21-27.

31. Bonita R, Beaglehole R. Recovery of motor function after stroke. Stroke. 1988;19:1497-1500.

32. Cincura C, Pontes-Neto OM, Neville IS, et al. Validation of the National Institutes of Health Stroke Scale, Modified Rankin Scale and Barthel Index in Brazil: the role of cultural adaptation and structured interviewing. Cerebrovasc Dis. 2009;27(2):119-122.

33. Lawton M, Brody E. Assessment of older people: selfmaintaining and instrumental activities of daily living. Gerontologist. 1969;9(3):179-186.

34. Wang CY, Hu MH, Chen HY, Li RH. Self-reported mobility and instrumental activities of daily living: test-retest reliability and criterion validity. J Aging Phys Activ. 2012;20(2): 186-197.

35. Tanaka JS. Multifaceted conceptions of fit in structural equation model. In: Long JS, Bollen KA, eds. Testing Structural Equation Models. Newbury Park: Sage; 1993:10-39.

36. Hu L, Bentler PM. Fit indices in covariance structure modeling: sensitivity to underparameterized model misspecification. Psychol Methods. 1998;3(4):424-453.

37. Hu L, Bentler PM. Cutoff criteria for fit indexes in covariance structure analysis: conventional criteria versus new alternatives. Struct Equ Modeling. 1999;6(1):1-55.

38. Browne MW, Cudeck R. Alternative ways of assessing model fit. In: Bollen KA, Long JS, eds. Testing Structural Equation Models. Newbury Park, CA: Sage; 1993:136-162.

39. Bandalos DL. The effects of item parceling on goodness-of-fit and parameter estimate bias in structural equation modeling. Struct Equ Modeling. 2002;9:78-102.

40. Nasser F, Takahashi T. The effect of using item parcels on ad hoc goodness-of-fit indexes in confirmatory factor analysis: an example using Sarason's Reactions to Tests. Appl Meas Educ. 2003;16:75-97.

41. McHorney CA, Tarlov AR. Individual-patient monitoring in clinical practice: are available health status surveys adequate? Qual Life Res. 1995;4(4):293-307. 
42. Apolone G, Mosconi P, Ware J. Questionario sullo stato di salute SF-36. Verona, Italy: Guerini e Associati; 1997.

43. Barbaranelli C. Analisi dei dati. 2nd ed. Milan, Italy: LED; 2007.

44. Munro BH. Statistical Methods for Health Care Research. 5th ed. Philadelphia PA: Lippincott Williams \& Wilkins; 2005.

45. Goncalves RS, Gil JN, Cavalheiro LM, Costa RD, Ferreira PL. Reliability and validity of the Portuguese version of the Stroke Impact Scale 2.0 (SIS 2.0). Qual Life Res. 2012; 21(4):691-696.

46. Combs SA, Dugan EL, Passmore M, et al. Balance, balance confidence, and health-related quality of life in persons with chronic stroke after body weight-supported treadmill training. Arch Phys Med Rehabil. 2010;91(12):1914-1919.

47. Bergstrom AL, Eriksson G, von Koch L, Tham K. Combined life satisfaction of persons with stroke and their caregivers: associations with caregiver burden and the impact of stroke. Health Qual Life Outcomes. 2011;9:1.

48. Marsden D, Quinn R, Pond N, et al. A multidisciplinary group programme in rural settings for community-dwelling chronic stroke survivors and their carers: a pilot randomized controlled trial. Clin Rehabil. 2010;24(4):328-341.

49. Shaughnessy M, Michael K, Resnick B. Impact of treadmill exercise on efficacy expectations, physical activity, and stroke recovery. J Neurosci Nurs. 2012;44(1):27-35.

50. Palmcrantz S, Holmqvist LW, Sommerfeld DK, Tistad M, Ytterberg C, von Koch L. Differences between younger and older individuals in their use of care and rehabilita- tion but not in self-perceived global recovery 1 year after stroke. J Neurol Sci. 2012;321(1-2):29-34.

51. Shamliyan TA, Bliss DZ, Du J, Ping R, Wilt TJ, Kane RL. Prevalence and risk factors of fecal incontinence in communitydwelling men. Rev Gastroenterol Disord. 2009;9(4): E97-E110.

52. Alvaro R, Araco F, Gravante G, et al. Epidemiological aspects of urinary incontinence in a female population of an Italian region. Int Urogynecol J Pelvic Floor Dysfunct. 2010;21: 873-883.

53. Raykov T, Marcoulides GA. An Introduction to Psychometrics. New York: Routledge, Taylor \& Francis Publishers; 2011.

54. Hobart JC, Williams LS, Moran K, Thompson AJ. Quality of life measurement after stroke: uses and abuses of the SF-36. Stroke. 2002;33(5):1348-1356.

55. Sarker SJ, Rudd AG, Douiri A, Wolfe CD. Comparison of 2 extended activities of daily living scales with the Barthel Index and predictors of their outcomes: cohort study within the South London Stroke Register (SLSR). Stroke. 2012;43(5): 1362-1369.

56. Simeone S, Savini S, Cohen MZ, Alvaro R, Vellone E. The experience of stroke survivors three months after being discharged home: A phenomenological investigation. Eur J Cardiovasc Nurs. In press. doi: 10.1177/1474515114522886.

57. Waltz C, Strickland O, Lenz E. Measurement in Nursing and Health Research. 4th ed. New York, NY: Springer Publishing Company; 2010. 